\title{
Detalhamento arquitetural do arcabouço estratigráfico Quaternário da plataforma sul fluminense, Bacia de Santos
}

\author{
Tardin, R.G.C.P., LAGEMAR-UFF; Reis, A.T., FAOC-UERJ; Silva, C.G., LAGEMAR-UFF
}

Copyright 2016, SBGf - Sociedade Brasileira de Geofísica

Este texto foi preparado para a apresentação no VII Simpósio Brasileiro de Geofísica, Ouro Preto, 25 a 27 de outubro de 2016. Seu conteúdo foi revisado pelo Comitế Técnico do VII SimBGt, mas não necessariamente representa a opinião da SBGf ou de seus associados. É proibida a reprodução total ou parcial deste material para propósitos comerciais sem prévia autorização da SBGf.

\section{Resumo}

A análise integrada de dados sísmicos de diferentes níveis de alta resolução permitiu um maior detalhamento da seção rasa ( 300 ms) da plataforma sul fluminense, entre a Baía de Guanabara e Cabo Frio. Até a realização deste trabalho, a seção rasa da plataforma fluminense era caracterizada como constituída essencialmente de 5 sequências principais de 100ka de duração (Sq1, Sq2, Sq2', Sq3 e Sq4), preservadas apenas como tratos de sistema regressivos desenvolvidos nos últimos $\sim 600 \mathrm{ka}$. Os resultados aqui obtidos permitiram a identificação de subunidades deposicionais intra-sequências Sq1, Sq2, Sq2', Sq3 e Sq4, evidenciando que tais sequencias são constituídas pela deposição e preservação de Tratos de Sistema Regressivos, Transgressivos e de Mar alto. Além disso, o detalhamento sísmico possibilitou também evidenciar que a sequência transgressiva depositada nos últimos 20 ka (unidade ainda em formação), ao contrário do que se acreditava, distribui-se de forma mais contínua ao longo de toda a plataforma continental da área de estudo.

\section{Introdução}

A plataforma continental sul fluminense, porção nordeste da Bacia de Santos, tem sido alvo de alguns estudos que focalizam o registro estratigráfico e os processos deposicionais do Quaternário Superior (e.g., Maia et al., 2010; Reis et al, 2011; Friederichs et al., 2013; Reis et al., 2013; Maia, 2014; Tardin, 2015) Esses estudos apontam que o empilhamento e arranjo arquitetural das sequências deposicionais quaternárias $(\sim 300 \mathrm{~ms}$ superficiais da sucessão estratigráfica) da área de estudo são condicionados, essencilamente, pelas oscilações glacio-eustáticas (Maia et al., 2010) orbitalmente induzidas (ciclos de Milankovitch de 100 ka de duração) responsáveis pelas sequências Sq1-Sq4 definidas por Maia et al. (2010). No entanto, muitos aspectos da história deposicional e da evolução estratigráfica da plataforma sul fluminense permanecem ainda pouco compreendidos, tais como o porquê da predominância apenas de unidades deposicionais regressivas. Além disso, trabalhos pretéritos, utilizando base de dados de resolução sísmica inferior à empregada no presente estudo, indicam que a sedimentação transgressiva que acompanhou a última deglaciação (sequência Sq5 de Maia et al., 2010), iniciada após o Último Máximo Glacial, estaria distribuída de forma esparsa ao longo da área de estudo, sendo pouco representativa em termos de espessura. Esta situação refletiria uma plataforma até então classificada como tipicamente faminta durante o Pleistoceno Superior-Holoceno (Zembruscki, 1979; Maia, 2009; Maia et al., 2010). Deste modo, o objetivo central deste estudo é detalhar a arquitetura interna das sequências Sq1-Sq5, através de uma base de dados ampliada (dados sísmicos de melhor resolução e testemunhagem rasa).

\section{Metodologia}

A identificação e interpretação das principais sequências sísmicas e seu significado ambiental e glacio-eustático utilizam metodologia baseada nos princípios da Sismoestratigrafia (Mitchum Jr. et al., 1977) e da Estratigrafia de Sequências de Alta Resolução (Catuneanu, 2006). A malha de dados sísmicos inclui dados de diferentes níveis de resolução (dados Sparker $700 \mathrm{~J}$, Boomer 200$300 \mathrm{~J}$ e duas fontes do tipo Chirp, uma com freqüências na faixa de $3,5-12 \mathrm{kHz}$ e outra de 0,5-12 kHz). O presente estudo utiliza também os resultados obtidos com a datação $\left({ }^{14} \mathrm{C}\right.$ AMS) e bioestratigrafia de testemunhos rasos, que contribuem para a caracterização das unidades de subfundo, especialmente para a definição do limite Pleistoceno Tardio-Holoceno (Figura 1).

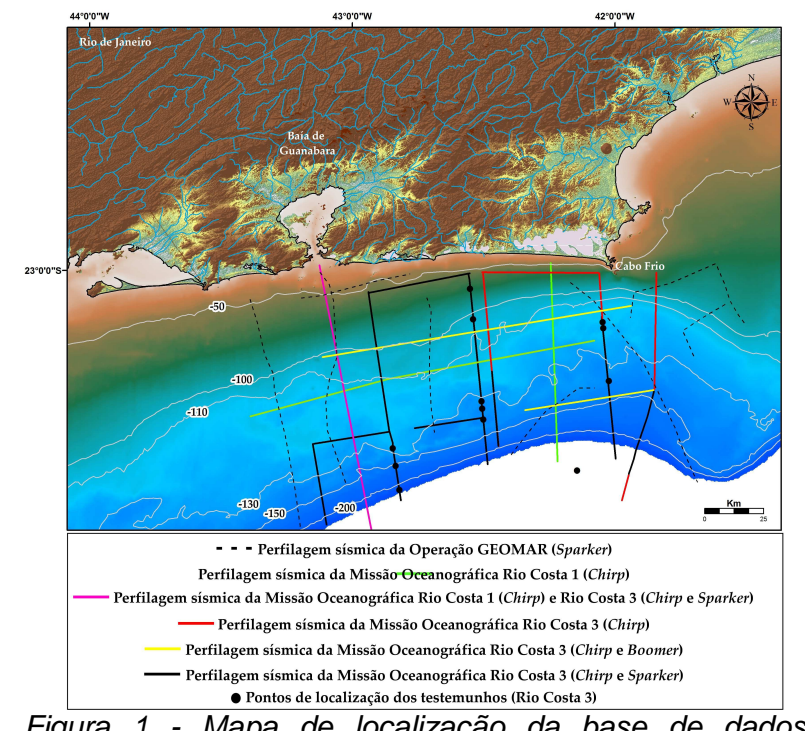

Figura 1 - Mapa de localização da base de dados sísmicos e dos testemunhos utilizados neste estudo. 


\section{Resultados}

No presente estudo, a seção que compõe os 300 ms superficiais da sucessão estratigráfica foi subdividida em unidades e subunidades limitadas em seu topo e base por discordâncias bem definidas e distribuídas de forma

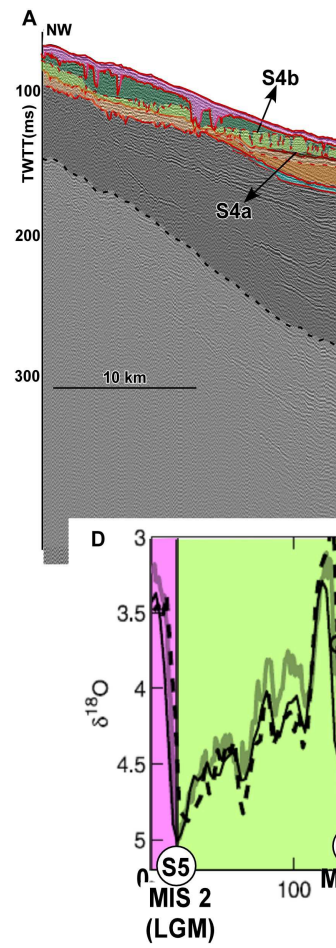

Figura 2 - (A) Exemplo de perfil sísmico interpretado (linha Se), ilustrando as superfícies que separam as principais sequências de Maia (2014) e as subsequências identificadas no presente estudo; (B) Correlação cronoestratigráfica entre as sequências e superfícies sísmicas interpretadas; (C) Associação com bibliografia existente (Maia, 2014); (D) Correlação entre as superfícies sísmicas identificadas e seu significado cronoestratigráfico e glacioeustático ao longo de compilação de curvas de variação do nível do mar (Siddall et al., 2003; Lisiecki e Raymo, 2005; Catuneanu, 2006). contínua em escala de toda a plataforma continental. A nomenclatura utilizada para tal é a mesma adotada por Maia (2009), Maia et al. (2010), Reis et al. (2013) e Maia (2014): sequências sísmicas Sq1, Sq2, Sq2', Sq3, Sq4 e Sq5, depositadas sobre as superfícies erosivas S1, S2, S2', S3, S4 e S5, respectivamente (Figura 2). 
Os mapas de isópacas das sequências sísmicas identificadas (Sq1-Sq5) resumem a distribuição regional das mesmas na área de estudo (Figura 3). A sequência sísmica Sq1, a mais antiga, distribui-se, majoritariamente, desde a isóbata de $-110 \mathrm{~m}$ até a região de borda de plataforma e início de talude. Sobre ela deposita-se a sequência Sq2, cujo surgimento acompanha a atual isóbata de $-130 \mathrm{~m}$. Acima desta, nas proximidades da atual isóbata de $-100 \mathrm{~m}$, uma terceira unidade encontrase depositada formando a sequência Sq2'. Por fim, as sequências $\mathrm{Sq} 3, \mathrm{Sq} 4$ e $\mathrm{Sq} 5$, empilham-se sobre as mais antigas, recobrindo toda a plataforma continental da área de estudo e configurando uma distribuição regional mais ampla e homogênea do que a observada nas sequências anteriormente descritas. De uma forma geral, todas as sequências sísmicas aqui identificadas, exceto a mais recente (Sq5, ainda em formação) formam pacotes que, de forma progressiva, tornam-se mais espessos em direção à bacia profunda, alcançando as maiores espessuras na região de quebra de plataforma e talude, expressos sob a forma de clinoformas progradantes.

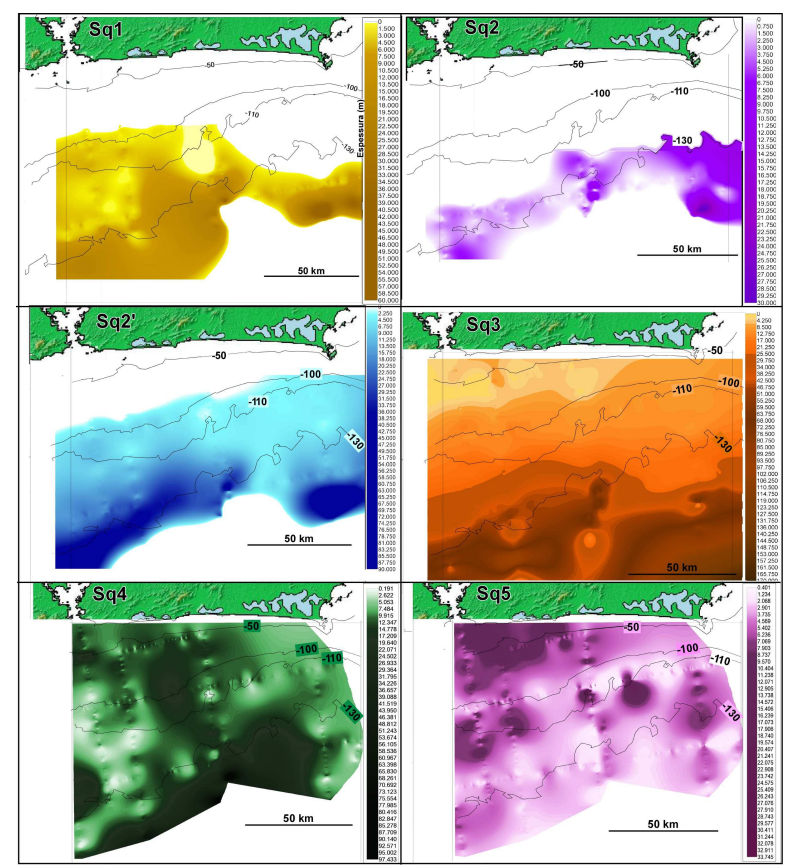

Figura 3 - Mapas de isópacas das 6 sequências sísmicas identificadas.

O melhor imageamento dos dados disponíveis permitiu a identificação de uma série de novas unidades sísmicas intra-sequências Sq1-Sq5, de diferentes tipos de fácies sísmicas e de um complexo conjunto de diferentes gerações de paleocanais (Figura 4). Dentre as fácies sísmicas encontradas, destacam-se clinoformas progradantes, tanto oblíquas-paralelas quanto oblíquastangenciais (COP e COT, respectivamente), prismas transgressivos, depósitos carbonáticos (bancos e pináculos) e diferentes tipos de fácies de preenchimento de canal fluvial (Figuras 4 e 5 ).

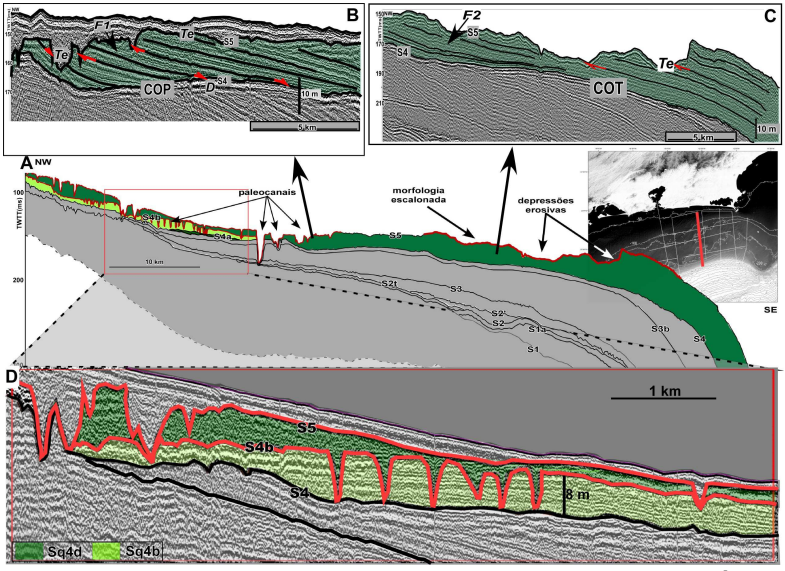

Figura 4 - Exemplo de interpretação da sequência sísmica Sq4 em perfil sísmico Sparker (A), destacando duas fácies sísmicas identificadas: $F 1$, em $B$, representando clinoformas oblíquas-paralelas (COP) e $F 2$, em $C$, constituindo clinoformas oblíquas-tangenciais (COT). Destaque para diferentes geraçôes de paleocanais $(D)$.

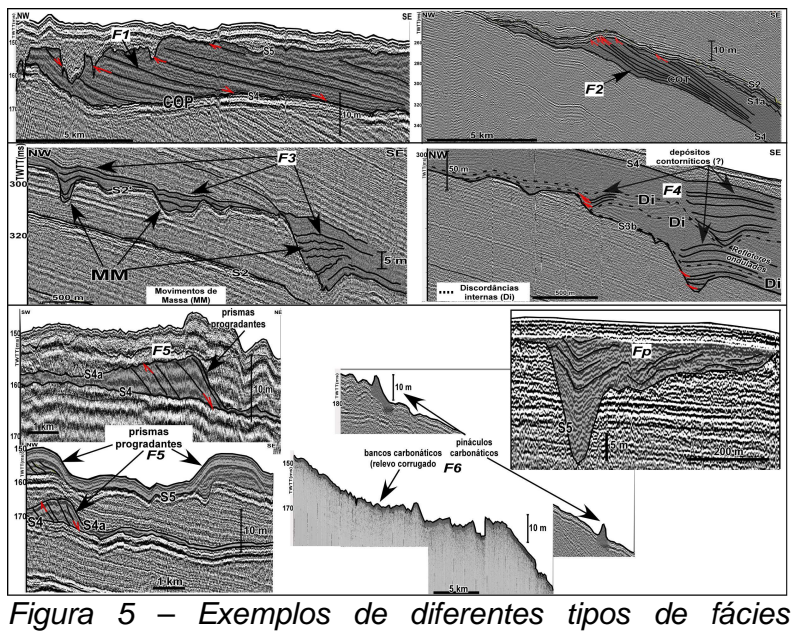
sísmicas identificadas no presente estudo.

\section{Discussão}

A interpretação da ciclicidade deposicional das sequências sísmicas identificadas, assim como o significado estratigráfico de suas superfícies limitantes, está de acordo com o proposto por Maia (2014). As superfícies S1, S2, S2', S3, S4 e S5 representam horizontes que evidencias a erosão desenvolvida durante máximos glaciais, e logo períodos de máxima exposição subaérea da plataforma continental (Figura 2). Desta forma, pode-se propor um significado cronoestratigráfico e glacio-eustático para as unidades intra-sequências Sq1- Sq5 identificadas no presente trabalho, baseado na integração de uma série de curvas de variações eustáticas globais, baseadas na razão isotópica de $\delta^{18} \mathrm{O}$ (Siddall et al., 2003; Lisiecki e Raymo, 2005; Rabineau et al., 2006; Figura 2) e nos resultados de datação e 
bioestratigrafia para as unidades mais superficiais, aqui disponibilizados (Figura 6).

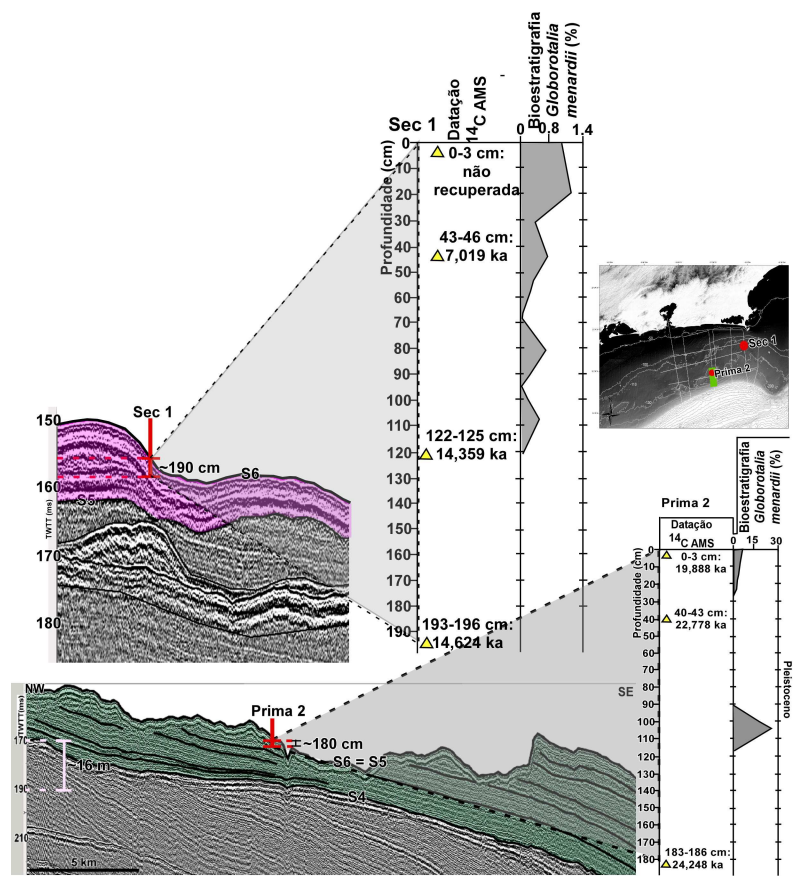

Figura 6 - Exemplos de correspondências entre interpretação sísmica e idades obtidas com os resultados de datação ( ${ }^{14} C$ AMS; LAC-UFF) e de bioestratigrafia (Laboratório de Sedimentologia da UFF, análises por Fabrício Ferreira) de dois testemunhos rasos adquiridos na área de estudo.

A sequência Sq1 formou-se entre 0.63 e 0.44 Ma (Maia et al., 2010). A subdivisão no presente estudo desta sequência nas unidades Sq1a e Sq1b evidencia, que a formação da sequência Sq1 ocorreu ao longo de dois ciclos com cerca de 100 ka de duração. Assim, as unidades Sq1a e Sq1b revelam fácies sísmicas (F1 e F2) caracterizadas por clinoformas progradantes que compõem prismas de regressão forçada (Falling Stage System Tract - FSST), observados, majoritariamente, na porção mais externa da plataforma continental (Figuras 2, 5 e 7).

A sequência Sq2 desenvolveu-se entre 0.44 e $0.35 \mathrm{Ma}$ (Maia et al., 2014) e, dentre as unidades observadas, é a que exibe o menor grau de preservação relativa. Os máximos glaciais que finalizam o ciclo deposicional da Sq2 indicam elevados valores de amplitude de queda eustática que podem justificar o alto grau de erosão observado nas superfícies discordantes S2 e S2', assim como a baixa preservação da sequência Sq2 (Rabineau et al. 2006; Figura 2). As sequências Sq2 e Sq2' também são compostas por prismas de regressão forçada distribuídos, principalmente, a partir da isóbata de $-130 \mathrm{~m}$ (fácies sísmica F2). A intensa erosão desencadeada durante os máximos glaciais do MIS 10 e do MIS 8 pode ser evidenciada pelas cicatrizes associadas a movimentos gravitacionais (fácies sísmica F3), observadas nas superfícies discordantes S2' e S3. Contudo, apesar dos fortes processos erosivos a que tais ciclos deposicionais foram submetidos, observa-se uma subdivisão destas sequências em quatro subunidades: Sq2t, Sq2't, Sq2r e Sq2'r. As duas primeiras são interpretadas como corpos transgressivos remanescentes, evidenciados pela fácies sísmica $F 5$ e preservados somente em alguns locais da plataforma continental da área de estudo (Figuras 5 e 7). Em um trabalho na mesma região, Maia (2009) identificou unidade transgressiva semelhante e atribuiu à sua superfície topo a característica de ravinamento.

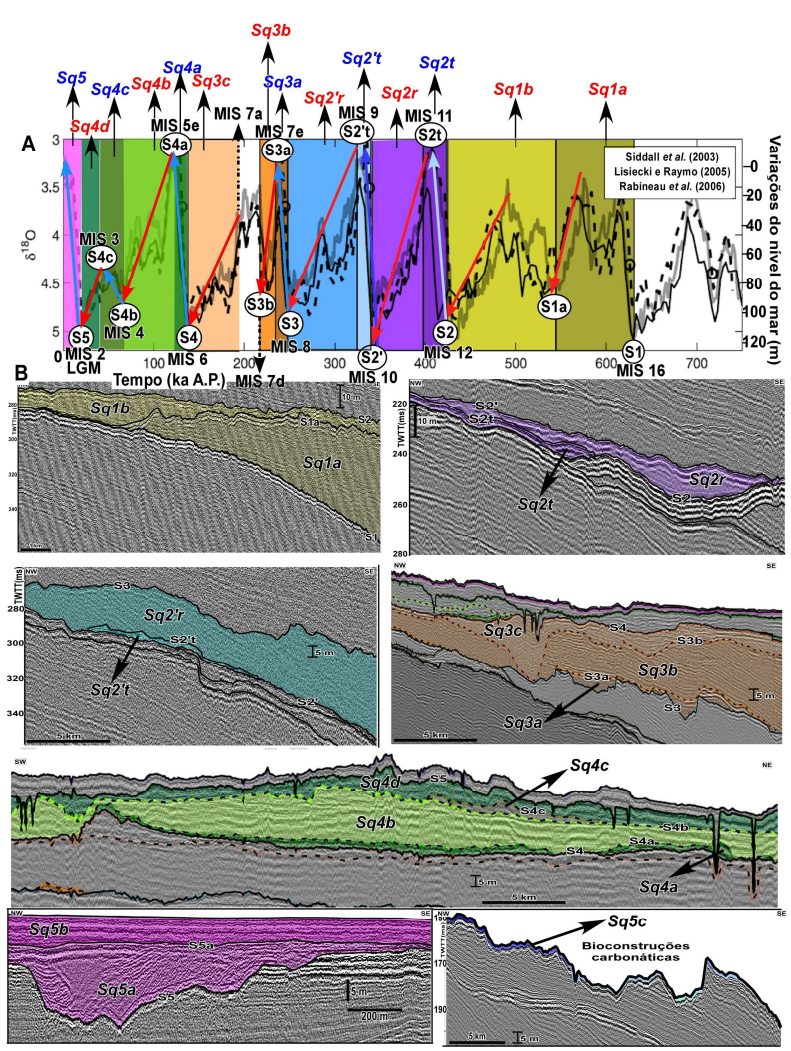

Figura 7 - (A) Correlação das sequências sísmicas e subunidades identificadas com seus respectivos ciclos de variação glacio-eustática. Curvas de variações eustáticas globais utilizadas, baseadas na razão isotópica de $\delta^{18} \mathrm{O}$ e bibliografia consultada: Siddall et al. (2003), Lisiecki e Raymo (2005) e Rabineau et al. (2006). (B) Exemplos das sequências sísmicas destacando as superfícies que as limitam, os horizontes internos que as subdividem e as subunidades formadas.

Entre 0.25 e $0.14 \mathrm{Ma}$ ocorreu a deposição da sequência sísmica Sq3 (Maia et al., 2010), compondo uma das sequências deposicionais mais espessas do arcabouço estratigráfico da plataforma continental da área de estudo (Figura 2). Entretanto, as curvas de variação eustática mostram que o ciclo associado à deposição desta sequência termina em um máximo glacial com alto valor de amplitude de queda eustática, o que resultaria em uma maior exposição subaérea da plataforma e, consequentemente, numa maior erosão e menor conservação da sequência Sq3. Dessa forma, uma 
possível explicação para tal contradição, também proposta por Maia (2009), seria um aumento da taxa de criação de espaço de acomodação durante o desenvolvimento da Sq3, o que promoveria a preservação de seus depósitos, mantendo-os espessos e distribuídos de forma contínua ao longo de toda a plataforma continental da região estudada. A primeira subseqüência observada, Sq3a estaria associada à deposição ocorrida durante a transgressão marinha desenvolvida entre o MIS 8 e o subestágio MIS 7e (Figura 7), preservada sobre a forma de fácies sísmicas típicas de transgressões marinhas (F5; Figura 5). As demais subunidades, $\mathrm{Sq} 3 \mathrm{~b}$ e Sq3c, exibem caráter regressivo, evidenciado por fácies sísmicas que formam clinoformas progradantes, típicas de períodos de regressão forçada (F1 e $\boldsymbol{F 2}$; Figuras 5 e 7). O topo da unidade $\mathrm{Sq} 3$, correspondente ao horizonte erosivo $\mathrm{S} 4$ intensamente erodido, é caracterizado pela presença de uma série de paleoincisões fluviais e também pela ocorrência de feições associadas à depósitos contorníticos, nas regiões de borda de plataforma e início de talude (fácies sísmica F4; Figuras 5 e 7).

A sequência $\mathrm{Sq} 4$ desenvolveu-se no período compreendido entre 0.14 e $0.018 \mathrm{Ma}$ (Maia et al., 2014; Figura 2). Esta unidade é a mais preservada dentre as demais. A idade Pleistoceno Superior do topo desta sequência pode ser comprovada pelos resultados das análises radiométricas e bioestratigráficas dos testemunhos rasos adquiridos neste trabalho. $O$ testemunho Prima-2, por exemplo, mostra idades que permitem o posicionamento cronoestratigráfico da Sq4 como representante do Pleistoceno Tardio (Figura 6). Assim como as demais sequências, a Sq4 também exibe horizontes discordantes internos que promovem a sua subdivisão em subunidades deposicionais: as subsequências Sq4a e Sq4c compostas por feições prismáticas e por fácies de preenchimento de canal típicas de eventos transgressivos ( $F 5$ e $\boldsymbol{F p}$; Figuras 5 e 7). Os prismas e depósitos transgressivos da sequência Sq4, também identificados por Marangoni et al. (2013) na vizinha bacia de Campos, distribuem-se por toda a plataforma continental da área de estudo, desde as regiões mais costeiras até a plataforma externa. Já as subunidades Sq4b e Sq4d são associadas a Tratos de Sistema de Regressão Forçada (Figuras 5 e 7). Ambas têm seus topos marcados por discordâncias bem delimitadas, caracterizadas por forte erosão e com uma série de incisões fluviais esculpidas em seus domínios. A subsequência Sq4d, também encontrada por Reis et al. (2013), corresponde à última grande queda eustática, ocorrida no período entre o final do MIS 3 e o MIS 2. O topo desta subunidade representa a discordância erosiva associada ao Último Máximo Glacial (LGM), superfície intensamente irregular, denominada S5. Tal intervalo temporal corresponde ao período de regressão forçada, evidenciado tanto através dos registros sísmicos ( $F 1$ e F2) quanto pelos resultados das análises radiométricas e bioestratigráficas dos testemunhos rasos do atual estudo (Figuras 5, 6 e 7).

Por fim, a sequência Sq5, encontra-se depositada sobre a superfície erosiva $\mathrm{S} 5$. Tal sequência, unidade ainda em construção, tem sua deposição associada à última transgressão marinha. A compilação da análise sismoestratigráfica com os resultados de datações absolutas e bioestratigrafia permite posicionar o limite temporal entre as sequências Sq4-Sq5 em 0.018 Ma (Figuras 2, 6 e 7). Além disso, trabalhos pretéritos baseados em dados de menor resolução (Maia, 2009; Maia et al., 2010) assumem que a Sq5 se distribui de forma esparsa ao longo da plataforma continental da área de estudo. Entretanto, os dados sísmicos de mais alta resolução aqui analisados evidenciam que tal unidade encontra-se distribuída de maneira contínua ao longo de toda a plataforma continental entre a Baía de Guanabara e Cabo Frio (Figura 3). Dentre as evidências do período de formação da sequência Sq5, as fácies e feições sísmicas como prismas costeiros, pacotes retrogradacionais e progradantes, dunas, fácies de preenchimento de canal e depósitos carbonáticos (Figuras 5 e 7) atestam que o desenvolvimento desta unidade se deu dominantemente em caráter transgressivo. Além disso, os resultados das análises radiométricas e bioestratigráficas em testemunhos rasos aqui realizadas também comprovam a natureza transgressiva dos depósitos pertencentes à Sq5. No testemunho Sec-1, por exemplo, os resultados de datação $\left({ }^{14} \mathrm{C}\right.$ AMS) mostram idades associadas à transgressão marinha iniciada, globalmente, após o LGM (Figura 6).

\section{Conclusões}

Os resultados obtidos recolocam a plataforma fluminense noutro contexto em termos de regime deposicional e nível de aporte sedimentar. A sucessão de tratos de sistema preservados intra-sequências $\mathrm{Sq} 1-\mathrm{Sq} 5$ evidencia uma plataforma continental não-faminta, durante os últimos 600 ka do Quaternário. A ampla distribuição sedimentar de depósitos pós-LGM (sequência Sq5) é evidência clara de aporte sedimentar moderno a esta plataforma, contrariando os modelos clássicos que atribuem apenas delgas e descontínuas camadas transgressivas plataformais, produto apenas do retrabalhamento de sedimentos reliquiares do Pleistoceno Superior.

\section{Agradecimentos}

Agradecimentos à CAPES, ao Convênio MCTI-Marinha do Brasil, à CPRM-Grupo de Geologia Marinha, à DHNMarinha do Brasil, ao LAGEMAR-UFF, ao Laboratório de Sedimentologia da UFF e ao Laboratório de Radiocarbono da UFF (LAC).

\section{Referências}

Catuneanu, O. 2006. Principles of Sequence Stratigraphy. Ed. Elsevier. 375p.

Friederichs, Y.L., Reis, A.T., Silva, C.G., Toulemonde, B, Guerra, J.V. 2013. Arquitetura Sísmica do Sistema Flúvio-estuarino da Baía de de Sepetiba Preservado na Estratigrafia Rasa da Plataforma Interna Adjacente, Rio de Janeiro. Revista Brasileira de Geociências, v. 43, n.1, p. 124-138. 
Lisiecki, L.E.; Raymo, M.E. 2005. A Pliocene-Pleistocene stack of 57 globally distributed benthic $\delta 180$ records: Pliocene-Pleistocene benthic stack. Paleoceanography, 20. doi:10.1029/2004PA001071.

Maia, R.M.C. 2009. Arcabouço estratigráfico e ciclicidade deposicional dos sistemas sedimentares plataformais ao largo do Estado do Rio de Janeiro, bacia de Santos. Dissertação (Mestrado em Geologia e Geofísica Marinha), Curso de Pós-Graduação em Geologia e Geofísica Marinha, Universidade Federal Fluminense, Rio de Janeiro. 127p.

Maia, R.M.C.; Reis, A.T.; Alves, E.C.; Silva, C.G.; Silva, A.; Guerra, J.V.; Gorini, C.; Silva, A.; Arantes-Oliveira, R. 2010. Architecture and stratigraphic framework of shelf sedimentary systems off Rio de Janeiro state, northern Santos basin-Brazil. Brazilian Journal of Oceanography, v. 58 (special issue, IGCP526), p. 15-29.

Maia, R.M.C. 2014. Evolução estratigráfica cenozoica da porção norte da bacia de santos: quantificação do fluxo sedimentar e da subsidência através de análise sísmica e de modelagem. Rio de janeiro. Tese de doutorado. Universidade Federal Fluminense.

Marangoni, M.B.; Reis A.T.; Silva, C. G.; Maia, R.M.C.; Guerra, J.V.; Arantes-oliveira, R.; Passos, M. M.; Bernardo, G.A. 2013. Arcabouço Estratigráfico e Ciclicidade Deposicional dos Sistemas Sedimentares Quaternários da Plataforma Sul da Bacia de Campos, RJ. Revista Brasileira de Geociências, v. 43, n.2, p. 285-300.

Mitchum JR., R.M.; Vail, P.R.; Sangree, J.B. 1977. Seismic Stratigraphy and Global Changes of Sea Level, Part 6: Stratigraphy Interpretation of Seismic Reflection Patterns in Deposicional Sequences. In: C. E. Payton (ed.) Seismic Stratigraphy - Applications to Hydrocarbon Exploration. Tulsa, AAPG, pp. 294-312 (Memoir \#26).
Reis, A.T.; Maia, R.M.C.; Silva, C.G.; Gorini, C.; Rabineau, M.; Alves, E.C.; Guerra, J.V.; Simoes, I.C.V.P.; Arantes-Oliveira, R. 2011. Feições geomorfológicas indicativas de variações eustáticas e de exposição subaérea da plataforma continental sul fluminense durante 0 pleistoceno superior-holoceno. Revista Brasileira de Geofísica, v. 29, n. 3, p. 1-24.

Reis, A.T.; Maia, R.M.C.; Silva, C.G.; Rabineau, M.; Guerra, J.V.; Gorini, C.; Ayres, A.; Arantes-Oliveira, R.; Benabdellouahed M., Simões, I.; Tardin, R. 2013. Origin of step-like and lobate seafloor features along the continental shelf off Rio de Janeiro State, Santos basinBrazil. Geomorphology. doi: 10.1016/j.geomorph.2013.04.037.

Siddall, M.; Rohling, E.J.; Almogi-Labin, A.; Hemleben, Ch.; Meischner, D.; Schmelzer, I.; Smeed, D.A. 2003. Sealevel fluctuations during the last glacial cycle. Nature 423, 853-858.

Tardin, R.G.C.P. 2015. Estratigrafia sísmica e paleodrenagem de sistemas deposicionais Quaternários da plataforma sul fluminense, Bacia de Santos. Dissertação de Mestrado - Universidade Federal Fluminense: Dinâmica dos Oceanos e da Terra; Área de Concentração: Geologia e Geofísica Marinha. Rio de Janeiro. Niterói. 278p.

Zembruscki, S.G. 1979. Geomorfologia da margem continental sul brasileira e das bacias oceânicas adjacentes. In: Hernani A. F. Chaves (Ed.), Relatório Final, Série Projeto REMAC. PETROBRAS/CENPES/DINTEP, Rio de Janeiro, 177p. 